\title{
A CLASS OF EXAMINATION OF FIBER ORIENTATION DEPENDENCY AND FAILURE WITH MULTI-SCALE SIMULATION
}

\author{
H. Sugiyama ${ }^{1}$ and S. Okazawa ${ }^{2}$ \\ ${ }^{1}$ Department of Mechanical Engineering, University of Yamanashi \\ 4-3-11 Takeda, Kofu, Yamanashi 400-8511, Japan \\ hirofumis@yamanashi.ac.jp \\ ${ }^{2}$ Department of Mechanical Engineering, University of Yamanashi \\ 4-3-11 Takeda, Kofu, Yamanashi 400-8511, Japan \\ sokazawa@yamanashi.acjp
}

Key words: Short Fiber Plastic, Multi-scale Simulation, Fiber Orientation, Strain Rate

\begin{abstract}
This paper examines the mechanical behavior of the short fiber reinforced plastic (SFRP) considering the microstructure using multi-scale simulation. The SFRP, which the microstructure consists of resin and fiver, has a rate, thermal, and fiber orientation dependency. The mechanical property is predicted by matrix and fiber properties. However, the properties by material testing are different from the prediction. Thus, this paper decides each microscopic material parameter on microstructure by the least-square method using the experimental data. Comparing the macro simulation results using homogenized values and the experimental results shows microstructure influences the macroscopic behavior. Moreover, the numerical examples compare the results under two types of tensile speed and three fiber orientation types. The present work completes the multi-scale simulation using a simple microstructure considering fiber orientation and strain rate.
\end{abstract}

\section{Introduction}

Due to social demands such as reducing environmental load and cost reduction, the transportation equipment industry is reducing the weight by replacing metal materials with resin materials. Among resin materials, those with improved mechanical properties by mixing carbon fiber and glass fiber at the molding time are called fiber reinforced plastics. Fiber reinforced plastics are characterized by high specific strength and specific rigidity, and their use is expanding in the industrial world. There are various types of base materials and fibers, and their characteristics are also diverse. Such composite materials, which are a combination of different materials, are devised to satisfy the desired mechanical properties. Composite materials such as fiber-reinforced resin materials, which improve strength by mixing fibers with the base resin, have excellent properties, as described above. Still, on the other hand, they have a complicated internal structure. It is difficult to grasp the properties of the microscopic structure (microstructure) of the material because it has. Therefore, in the past, the material properties have been grasped by the approach from the material test. However, the material properties obtained from the material test are obtained only from the macroscopic structure (macrostructure). The properties of the microstructure are not fully considered. Besides, the constitutive law used in conventional CAE does not consider the 
microstructure of composite materials, and analyzes using material properties based on macrostructures have been performed. On the other hand, there is multi-scale modeling to model phenomena based on the homogenization method [1]-[7]. In multi-scale modeling, by deriving the material properties of the macrostructure through the analysis of the microstructure of the material, it is possible to analyze the micro and macro phenomena in a coupled manner. Therefore, multi-scale analysis is used to predict mechanical properties considering microstructure. Here, fiber reinforced plastics are known to have fiber orientation dependence, velocity dependence, and temperature dependence. It is not realistic to obtain all of them from experiments, so modeling numerical simulation is required.

Based on this, the purpose of this study is to evaluate the mechanical properties of materials by multiscale analysis focusing on fiber orientation and velocity dependence for limited material test results. By comparing the experimental results of the tensile test with the analytical values, the influence of the microstructure characteristics on the velocity dependence and fiber orientation dependence, which are the characteristics of resin materials, will be investigated. After explaining the multi-scale analysis, the microstructure model and the handling of fiber orientation are described. In the numerical analysis example, the macroscopic changes in the behavior of the material and the influence of the microstructure conditions are compared and examined in the deformation region where the nonlinearity is not strong.

\section{Multi-sclae modeling for SFRP}

\subsection{Multi-scale simulation based on homogenization}

A multi-scale analysis is a method for approximate analysis of a two-variable boundary value problem based on nonlinear homogenization theory yhile naintaining both micro and macro coupled effects. It
is assumed that the smallest structural unit called unit cell is periodically and repeatedly arranged in
the object's microscopic area. It is assuned that the entire structure is covered with minute unit cells.
The characteristics of the macrostructure are defined by the geometric shape and material properties in
the unit cell. Numerical material tests are performed on unit cels with material properties set, and macro mechanical properties are obtained using the obtained micro response. In the commercial code used, a for numerical material tests while following multi-scale analysis based on the general homogenization method [1][8].

\subsection{Unit cell geometry}

In this paper, we consider the unit cell consists of matrix and fiber. First, a unit cell, which is the smallest unit of the periodic repeating structure of the material, is created. In this paper, it is assumed that the fiber in the matrix is short. Therefore, we assume that the fibers are discontinuous. In other words, the fibers are treated as fully included in the matrix. A schematic diagram of the unit cell model is shown in Figure 1. Figure 1 shows that the fiber is the cylinder and the matrix is hexahedral. Thus, the fiber in the unit cell, looking at the $\mathrm{x}$-axis direction, is shorter than the matrix's ends. The parameters used to create the unit cell are shown in Table 1. The microstructure generates based on each parameter on the Table 1 and volume fraction. 

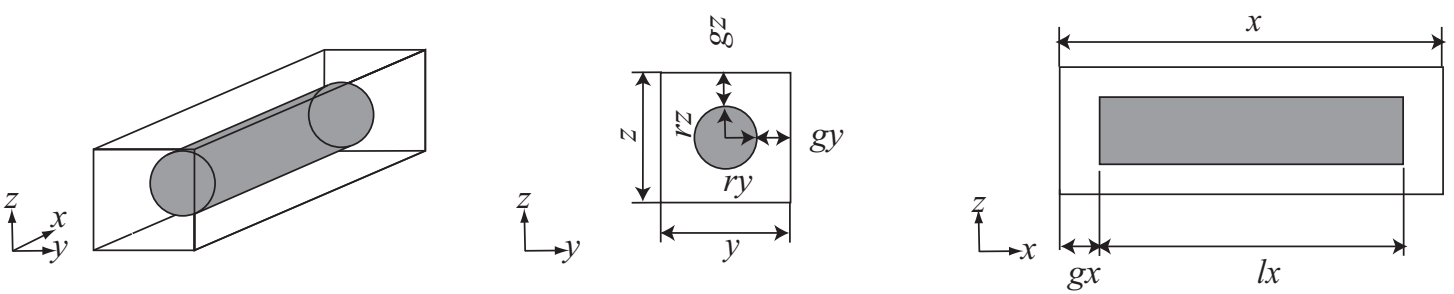

Figure 1: Illustration of unit cell model

Table 1: Parameter of unit cell

Parameters

Matrix length of $\mathrm{x}$-direction $x$

Matrix length of z-direction $y$

Matrix length of z-direction $z$

Fiber length of $\mathrm{x}$-direction $\quad l_{x}$

Fiber raius of $y$-direction

Fiber raius of z-direction

Gap of x-direction

Gap of y-direction

Gap of z-direction

Table 2: Parameters of matrix and fiber

\begin{tabular}{ccc} 
& Matrix & Fiber \\
\hline Young modulus[MPa] & $E_{m}$ & $E_{f}$ \\
Poisson's ratio & $v_{m}$ & $V_{f}$
\end{tabular}

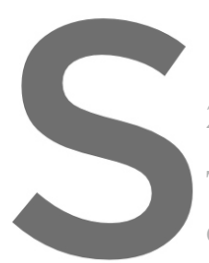

2.3 Inelastic model

The mechanical property of the fiber shows elastic of the matrix shows inelastic
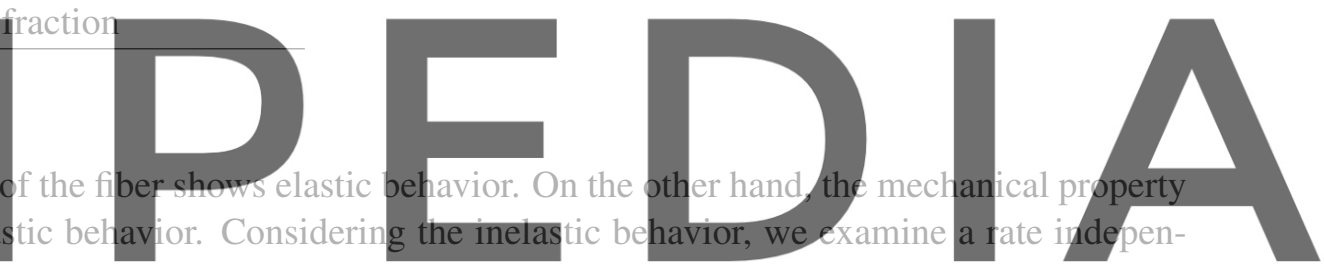

dent elasto-plastic model. The hardening function of the rate independent model is expressed as the

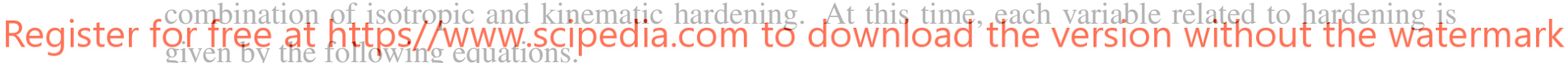

$$
\begin{gathered}
h(\alpha)=\left(K_{\infty}-K_{0}\right)[1-\exp (-\delta \alpha)]+H^{\prime} \alpha \\
K(\alpha)=K_{0}+\theta h(\alpha) \quad \theta \in[0,1] \\
H(\alpha)=(1-\theta) h(\alpha) \quad \theta \in[0,1]
\end{gathered}
$$

Here, the equation (1) expresses the curing law to be used. The equation (2) and equation (3) represent isotropic curing and transfer curing, respectively. At this time, $K_{0}$ is the initial yield stress, $K_{\infty}$ is the tensile strength, $\alpha$ is the cumulative plastic strain, $\delta$ is the exponential part of the evolution law, and $\theta$ is the transfer hardening. And represents the rate of isotropic hardening. Moreover, Table 2 shows the basic linear parameters of matrix and fibers. $E_{m}$ is the Young modulus for matrix, $E_{f}$ is the Young modulus for fiber, $v_{m}$ is the Poisson's ratio for matrix and $v_{f}$ is the Poisson's ratio for fiber, respectively. 


\subsection{Fiber orientation tensor}

In order to consider the dependency of the fiber orientation, each angle is defined. Figure 2 shows illustration of the fiber orientation.

$$
\left(\begin{array}{c}
\xi \\
\eta \\
\theta
\end{array}\right)(\xi \eta \theta)=\left[\begin{array}{ccc}
\xi^{2} & \xi \eta & \xi \theta \\
\eta \xi & \eta^{2} & \eta \theta \\
\theta \xi & \theta \eta & \theta^{2}
\end{array}\right]
$$

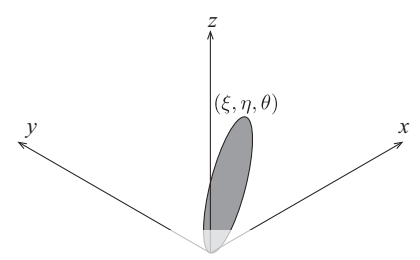

Figure 2: Definition of the fiber direction

This can be expressed using the fiber orientation tensor $a_{i j}$, which is handled in the actual resin flow analysis, as shown in the following equation.
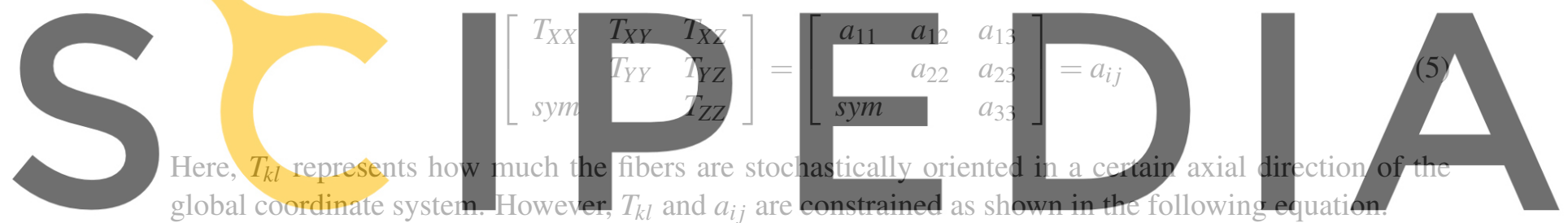

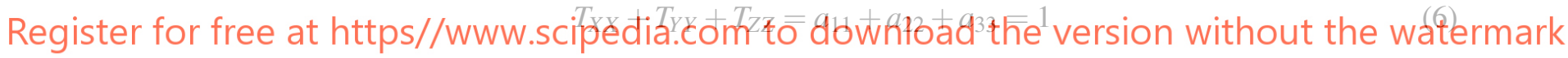

Since $T_{X X}$ stochastically represents how many fibers are oriented in the $x$ axis direction of the global coordinate system, The fiber orientation tensor when the fibers are actually oriented in the $x$ axis direction is obtained as follows.

$$
\left(\begin{array}{l}
a \\
b \\
c
\end{array}\right)\left(\begin{array}{l}
\mathrm{a} b \mathrm{c} c \\
0
\end{array}\right)=\left(\begin{array}{l}
1 \\
0 \\
0
\end{array}\right)\left(\begin{array}{lll}
1 & 0 & 0
\end{array}\right)=\left[\begin{array}{lll}
1 & 0 & 0 \\
0 & 0 & 0 \\
0 & 0 & 0
\end{array}\right]=a_{i j}
$$

When using the fiber orientation tensor, $T_{X X}, T_{Y Y}, T_{Z Z}, T_{X Y}, T_{Y Z}, T_{X Z}$ The fiber orientation can be reflected in the analysis by creating an input file corresponding to each element number of the value.

\section{Tensile testing}

The multi-scale analysis is performed on the shape of the material test piece used in the tensile test. By comparing and verifying the experimental results of the tensile test of the short fiber reinforced plastic PA66GF30 and the analysis results of the model created using multi-scale modeling, we will examine the modeling that can reproduce the mechanical properties of the target material. 


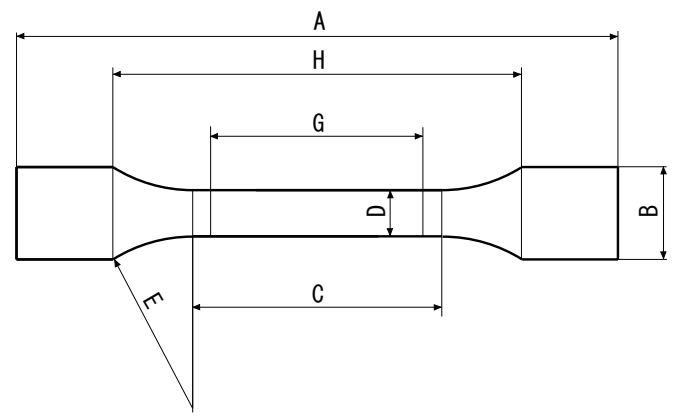

Figure 3: Testpiece

Table 3: Parameters of test piece

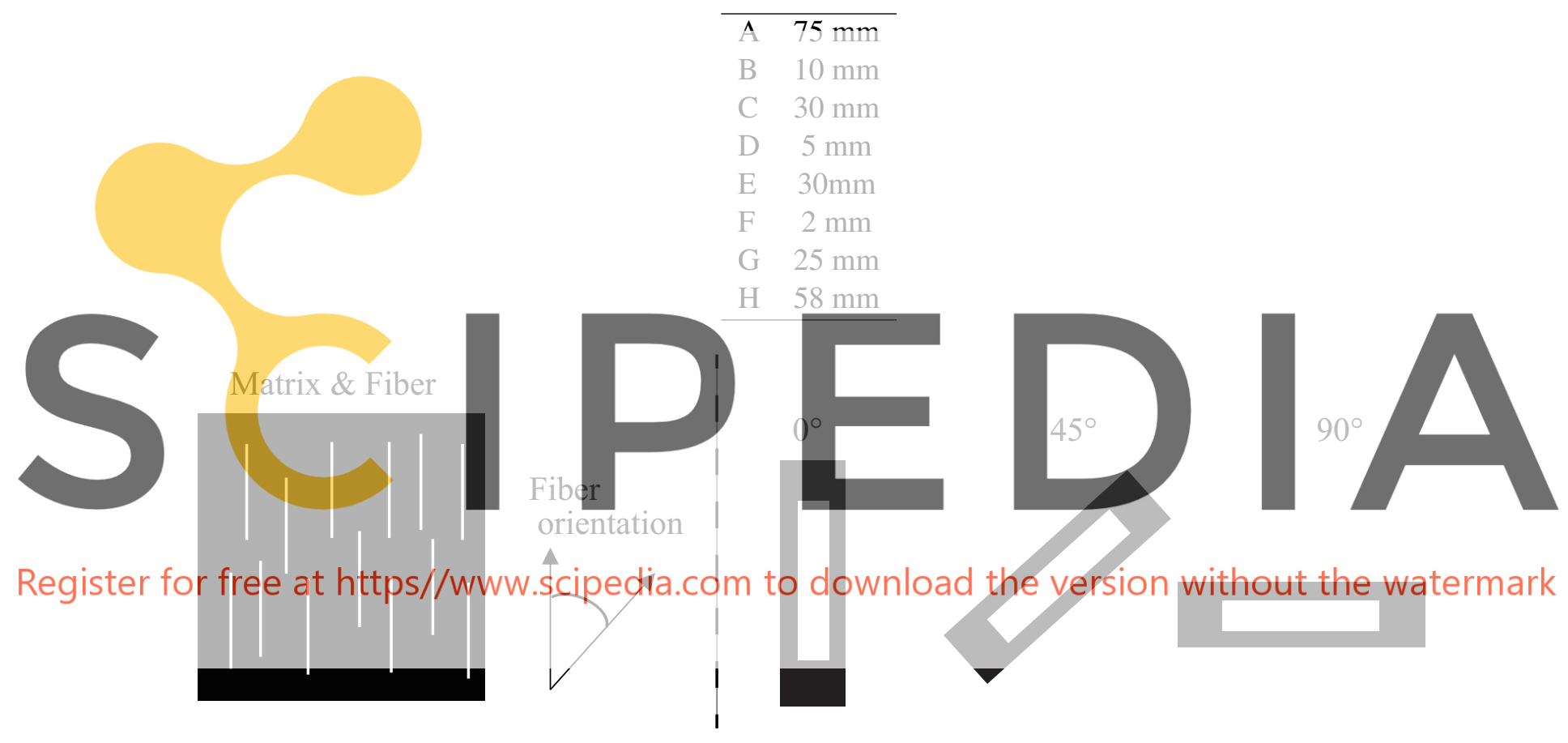

Figure 4: Fiber orientation

\subsection{Experimental procedure}

In the tensile testing, two loading conditions and three fiber orientations are conducted. The tensile rate are $5 \mathrm{~mm} / \mathrm{min}$ and $100 \mathrm{~mm} / \mathrm{min}$ and three conditions of fiber orientation are $0^{\circ}, 45^{\circ}$ and $90^{\circ}$. A schematic diagram of the tensile test piece is shown in Figure 3. Each length of the test piece expresses in Table 3. The experiment assumes that each test piece has a uniform fiber orientation in the set direction. Figure 4 shows an illustration of the fiber orientation. In this case, fiber direction is assumed a two-dimensional situation. A strain gauge measures the tensile direction strain, and the force is measured by the load cell. Therefore, the nominal stress/strain is calculated by using each measured value. 


\subsection{Numerical simulations}

As shown in Figure 5, the 1/4 model is modeled as the analysis model considering the symmetry of the test piece. Eight-node Hexahedral solid element meshes are employed. Furthermore, the boundary conditions are given as shown in Figure 5. The unit cell shape is based on the Figure 1, and Table 1 shows the unit cell size. Secondly, we consider the case where the parameter fitting of the material constitutive law. In this case, parameter fitting is performed by the least squares method in the microstructure using the experimental data of the tensile velocity of $5 \mathrm{~mm} / \mathrm{min}$ at the fiber orientation of $0^{\circ}$. At this time, the equation $(1) \sim(3)$ is used as the constitutive law of the fiber and the matrix. In order to conduct the numerical simulation for the microstructure, the initial value of the linear characteristics of matrix and fiber is set using the general value of each material. And then, the elastic modulus $E_{f}$ of the fiber was calculated with the initial slope of the stress-strain curve based on the experimental data. Table 5 shows the fitting result. As is clear from Table 5, the value is significantly smaller than the reference value. This is thought to be because the target fiber is short. Thus, the way the load is applied and the handling of rigidity inside the actual materialize different from the reference value assumption. There is room for consideration in measuring the internal structure's rigidity, but here we adopt this fitted parameter. Next, Table 6 shows the results of fitting the elasto-plastic constitutive parameters of the matrix. Here, $\beta_{1}$ and $\beta_{2}$ are parameters for considering the influence of the volumetric strain on the plastic deformation of the plastic, respectively. In this analysis, these were also adopted for fitting as one of the parameters. Multiscale simulation is performed using the microstructure parameters of Table 5 and 6 . The macrostructure simulation using the different fiber orientations and two types of tensile rate was conducted, and the

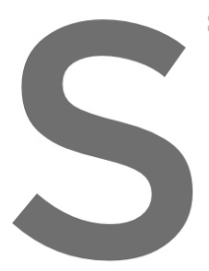
simulation results were
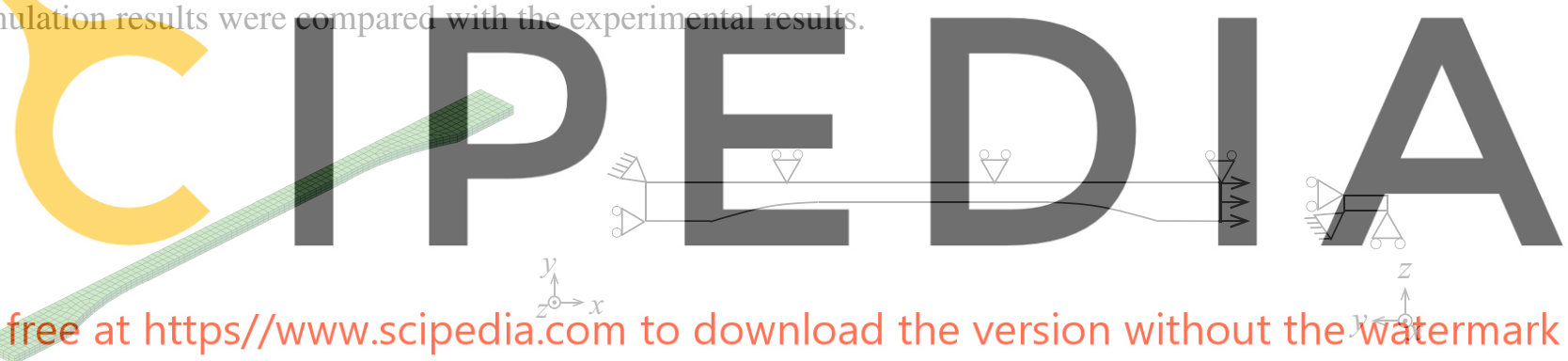

Register for free at https//www.scipedia.com to download the version without the katermark

Figure 5: Boundary condition

Table 4: Parameters of Unit cell

\begin{tabular}{cc}
\hline Matrix length of x-direction & 17.8256 \\
Matrix length of z-direction & 1.0 \\
Matrix length of z-direction & 1.0 \\
Fiber length of x-direction & 16.2051 \\
Fiber raius of y-direction & 0.3241 \\
Fiber raius of z-direction & 0.3241 \\
Gap of x-direction & 0.08103 \\
Volume fraction [\%] & 30 \\
\hline
\end{tabular}


Table 5: Optimized parameter

\begin{tabular}{lcc}
\hline & Reference & Fitted value \\
\hline Young modulus of fiber $E_{f}[\mathrm{MPa}]$ & 72395.0 & 16584.2 \\
\hline
\end{tabular}

Table 6: Parameters of matrix

\begin{tabular}{cc}
\hline Parameters & Fitted values \\
\hline$K_{y}[\mathrm{MPa}]$ & 19.62 \\
$K_{\infty}[\mathrm{MPa}]$ & 66.27 \\
$\delta$ & 100.5 \\
$H$ & $-4.0 \times 10^{-4}$ \\
$\theta$ & $1.2 \times 10^{-3}$ \\
$\beta 1$ & $7.5 \times 10^{-5}$ \\
$\beta 2$ & $-9.99 \times 10^{-7}$ \\
\hline
\end{tabular}

Figure 6 and Figure 7 show the results of stress-strain curves in macrostructures with tensile velocities of $5 \mathrm{~mm} / \mathrm{min}$ and tensile velocities of $100 \mathrm{~mm} / \mathrm{min}$, respectively. Figure 6 shows that the present results correctly represent deformation in almost all strain ranges. The magnitude relation of stress due to the difference in orientation angle matches the experimental result. However, it is necessary to study where the peak of stress appears in the simulation as the deformation becomes larger. Besides, it is necessary to consider the effect on the part where stable results are not obtained due to uncertainty, although three experimental results were carried out.

On the other hand, it is clear that the present result under the tensile speed of $100 \mathrm{~mm} / \mathrm{min}$ in Figure 7 is different from the previous result. When the orientation is $0^{\circ}$, the simulation result is close to the experimental result near the strain of $1 \%$ and $3 \%$, but the error is large in the region where $2 \%$. Here, it

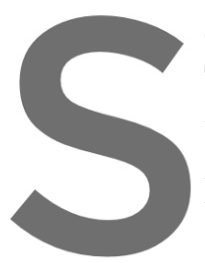
can be seen that the stress The main reason for sinu the case where the tensile From the numerical sinulations, the fiber orientation a simulation. The material
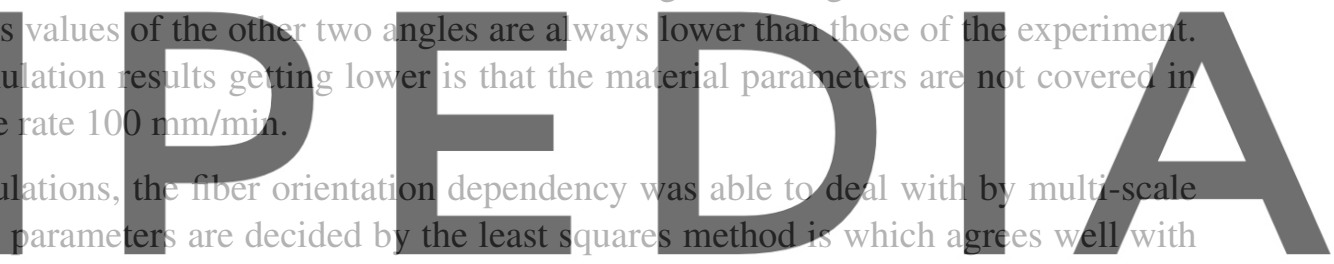

the experimental result. The results of the lower tensile rate case well match.

Register for free at https//www.scipedia.com to download the version without the watermark

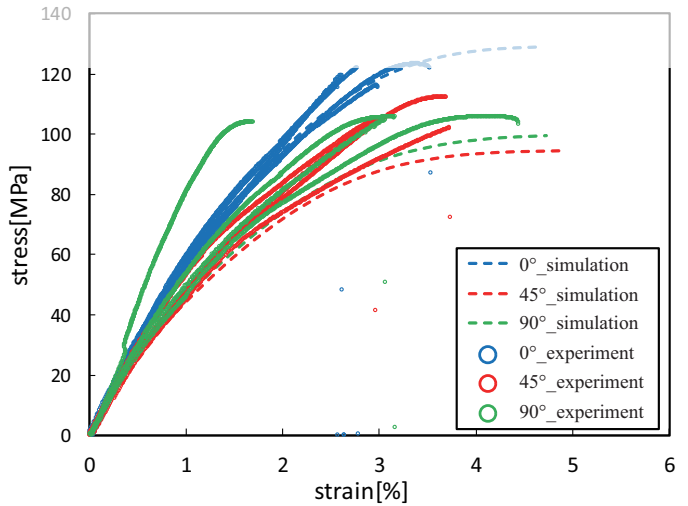

Figure 6: $5 \mathrm{~mm} / \mathrm{min}$ : Stress-strain curve

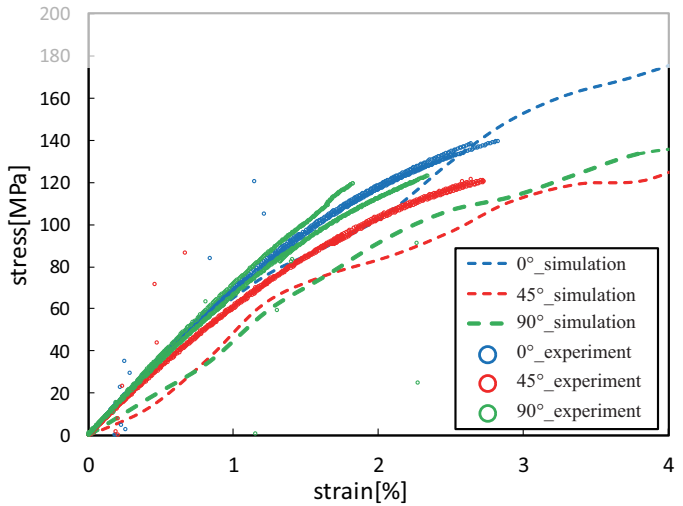

Figure 7: 100mm/min: Stress-strain curve 


\section{Conclutions}

In this paper, we performed multi-scale analysis on short fiber reinforced plastics and obtained the following findings.

- Parameter fitting estimates the modulus of elastic and inelastic model

- Response of the macrostructure can be well expressed with respect to the tensile speed with parameter fitting

- The lower displacement rate simulation well matches

- The higher displacement rate simulation has errors. The difference in behavior at tensile speeds of $100 \mathrm{~mm} /$ min could not be fully expressed.

The following can be considered as future issues.

- Further consider the selection of target values for parameter fitting

- Considering the material model considering the rate dependency

- Introduce a model that can consider hardening such as fiber peeling for microstructure

\section{REFERENCES}

[1] Yuan, Z. and Fish, J. Hierarchical model reduction at multiple scales, Int. J. Num. Meth. Engng. (2009) 79:314-339.
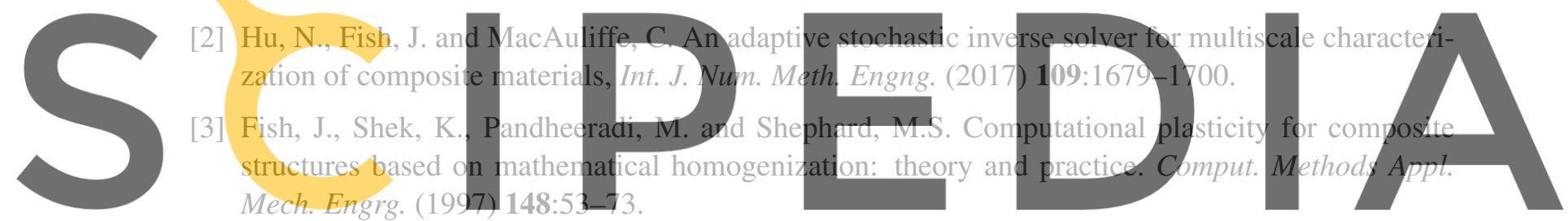

[4]. Fish, J. and Shek, K.L. Finite deformation plasticity of composite structures: computational models

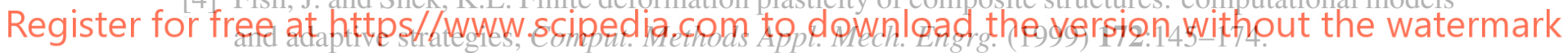

[5] Terada, K., Saiki, I., Matsui, K. and Yamakawa, Y. Two-scale kinematics and linearization for simultaneous two-scale analysis of periodic heterogeneous solids at finite strain, Comput. Methods Appl. Mech. Engrg. (2003) 192: 3531-3563.

[6] Terada, K. and Kikuchi, N. A class of general algorithms for multi-scale analysis of heterogeneous media. Comput. Methods Appl. Mech. Engrg. (2001) 190:5427-5464.

[7] Matsui, K., Terada, K. and Yuge, K. Two-scale finite element analysis of heterogeneous solids with periodic microstructures. Computers and Structures (2004) 82(7-8):593-606.

[8] Altair. Multiscale Designer, 2019. (Available from: http://www.altairhyperworks.com/product/MultiscaleDesigner) [Accessed on 1 February 2021]. 